DOI: 10.1002/adma.((please add manuscript number))

Submitted to

Article type: Communication

\title{
Peptide-Tunable Drug Cytotoxicity via One-Step Assembled Polymer Nanoparticles
}

Kang Liang, Joseph J. Richardson, Hirotaka Ejima, Georgina K. Such, Jiwei Cui, and Frank Caruso*

((Optional Dedication))

K. Liang, J. J. Richardson, Dr. H. Ejima, Dr. G. K. Such, Dr. J. Cui, Prof. F. Caruso Department of Chemical and Biomolecular Engineering, The University of

Melbourne, Parkville, Victoria 3010 (Australia)

E-mail: fcaruso@unimelb.edu.au

Keywords: (self-assembly, co-delivery, drug delivery, peptide, biomaterials)

The delivery of therapeutics into cells is important for biomedical applications ranging from gene therapy to drug delivery. Since the commercial introduction of Doxil $^{\circledR}$, particle-based drug carriers have been used to improve therapeutic outcomes. ${ }^{[1]}$ Carriers are useful for transporting drugs to cells and reducing the nonspecific toxicity of the drug, while cocktails of therapeutics are receiving intense study for enhancing the specific desired toxicity. ${ }^{[2]}$ The combination of a non-toxic facilitating molecule with specific therapeutics has proven to be especially useful for promoting enhanced cellular responses. ${ }^{[3]}$ Cell penetrating peptides (CPPs) are a useful class of facilitator molecules that have found application in imaging, and gene and drug delivery. ${ }^{[4]}$ A particularly interesting CPP is the non-toxic arginine-rich trans-activating transcriptional activator (Tat) and the corresponding engineered derivatives. These CPPs have been used to enhance cellular uptake, endosomal escape and/or nuclear targeting to facilitate improved therapeutic responses. ${ }^{[3 a, 5]}$ 
Submitted to

\section{ADVANCED}

Although co-delivery of a CPP and a drug is widely used in research, no reports have shown tunable cytotoxicity by varying the ratio of CPP to drug, which would allow enhanced control over dosage. The lack of studies into peptide-gated cytotoxicity is most likely limited because CPPs are often conjugated directly to the carrier or cargo, thereby making it difficult to selectively vary the concentration of one or the other. $^{[3 b, 4 b, 6]}$ Herein, we report for the first time the tunable co-delivery of an engineered Tat-based CPP and a chemotherapeutic drug for enhanced and controllable cytotoxicity using an engineered polymer carrier assembled in one-step. The cytotoxicity was selectively tuned up to a factor of 30 simply by varying the amount of CPP loaded into the nanoparticles (NPs). Monodisperse, multi-cargo loaded NPs were assembled through a modular one-step process by mixing two simple polymer molecules; a polyethylene glycol-doxorubicin conjugate (PEG-Dox), and a poly(2-diisopropylaminoethyl methacrylate) (PDPA) homopolymer, with a custom designed arginine (R) and histidine (H) containing CPP; $\mathrm{NH}_{2}-\mathrm{HHHH}-\mathrm{RRRR}-$ $\mathrm{COOH}(\mathrm{H} 4 \mathrm{R} 4)$.

H4R4 was designed to combine the benefits of arginine/Tat-based CPPs with the endosomal escape and $\mathrm{pH}$-dependent properties of histidine. ${ }^{[7]}$ With a pKa of $\sim 6.5,{ }^{[8]}$ poly-histidine has similar material characteristics to PDPA; that is, both shift from hydrophobic to hydrophilic when the $\mathrm{pH}$ is below $~ 6.5 .^{[9]}$ Therefore, H4R4 selfassembled with PDPA and PEG-Dox to form monodisperse NPs. At physiological pH, PDPA forms a hydrophobic core, immobilizing H4R4 and Dox into place while allowing PEG to extend out from the core and to form a hydrophilic corona. Following entrance into the acidic endosomal compartments, ${ }^{[10]}$ PDPA and H4 become protonated and destabilize the NPs due to electrostatic repulsion, thereby 
Submitted to

releasing Dox (Scheme 1). PDPA was chosen as a core stabilizer because of its $\mathrm{pH}$ responsive nature. Our previous work has shown that $\mathrm{pH}$-sensitive PDPA-based systems can be internalized and disassembled in cells. ${ }^{[11]}$ Furthermore, PDPA eliminates the leakage and concentration-dependent stability issues of some other nanostructures (e.g., non-cross-linked micelles, polymersomes). ${ }^{[12]}$ The reported NPs offer three other major advantages over similar systems. First, while most NP- and micelle-based systems have reduced toxicity in comparison to free drug, H4R4 allowed our system to have a significantly higher toxicity than free drug. Second, the fact that the peptide and anti-cancer drug are unconjugated allows for a high degree of control over peptide loading and enhanced chemotherapeutic efficacy. Third, the therapeutic cargo was prepared through a one-step reaction that only requires simple conjugation chemistry rather than complex polymer synthesis. This strategy could be easily applied to a wide range of biomolecules. This simple and modular assembly technique will allow different hydrophobic therapeutic molecules to be easily incorporated, making it attractive for diverse biomedical applications. The prepared NPs encompass pH-induced loading and release of cargo, and achieve simultaneous and tunable co-delivery of peptides and anticancer drugs.
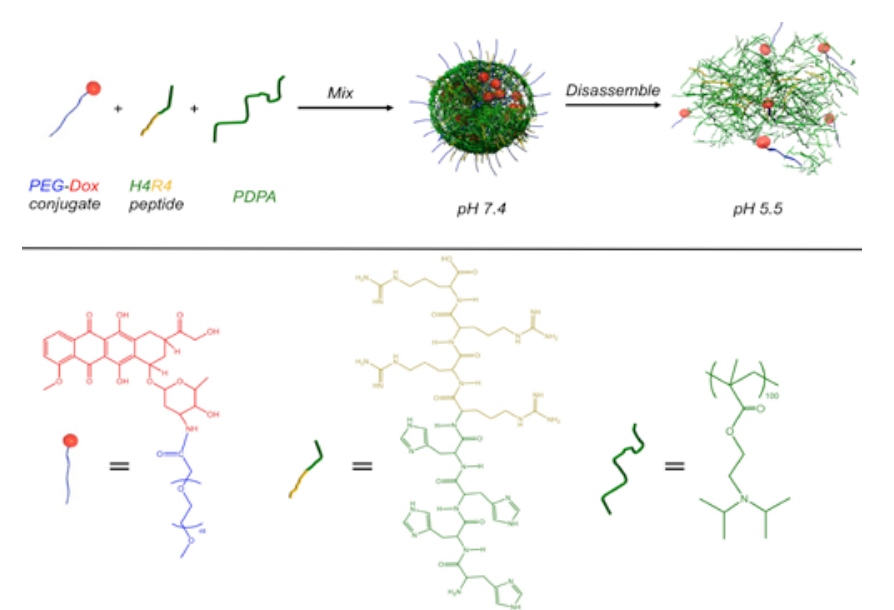

Scheme 1. Schematic illustration showing the co-assembly of Dox and H4R4-loaded NPs at pH 7.4 and disassembly upon a decrease in $\mathrm{pH}$. 


\section{Submitted to

The self-assembly of drug and peptide loaded NPs (denoted PDPA NPs and H4R4PDPA NPs as NPs without and with the loading of H4R4, respectively) is illustrated in Scheme 1. PDPA was synthesized via atom transfer radical polymerization (ATRP), while Dox was conjugated to PEG $\left(\mathrm{M}_{\mathrm{w}} 2.3 \mathrm{kDa}\right)$ through the reaction between $N$-hydroxysuccinimide esters (NHS) at the PEG terminal and the amine in the Dox molecule. The conversion of the amine likely causes the Dox portion of PEGDox to become more hydrophobic, allowing it to associate with PDPA at physiological pH. A co-solvent evaporation method was employed in synthesizing the NPs. PEG-Dox, PDPA, and H4R4 were dissolved in ethanol at a mass ratio of 2:1:0.1 (3.0 wt\%), respectively, and transferred into phosphate buffered saline (PBS) at $\mathrm{pH}$ 7.4 while stirring. Following the evaporation of ethanol, the solution was passed through filters $(0.45 \mu \mathrm{m})$ to remove large aggregates and dialyzed against PBS to remove excess free molecules. Transmission electron microscopy (TEM), atomic force microscopy (AFM) (Figure 1), and dynamic light scattering (DLS) (Supporting Information, Figure S1) revealed the formation of NPs (polydispersity index, PDI 0.04) with an average size of roughly $100 \mathrm{~nm}$, irrespective of H4R4 loading (PDI 0.17 for $3.0 \mathrm{wt} \%)$. No defined structures were obtained in the absence of either PDPA or PEG-Dox, indicating that both of these molecules are critical for the NP formation. NPs with a size of $100 \mathrm{~nm}$ are advantageous for drug delivery because they are capable of utilizing the enhanced permeability and retention (EPR) effect. ${ }^{[13]}$ Furthermore, Fourier transform infrared spectroscopy (FTIR) and matrix-assisted laser desorption/ionization-time of flight (MALDI-TOF) spectrometry (Supporting Information, Figures S2 and S3) were used to confirm the incorporation of PEG-Dox and H4R4 in the NPs. From FTIR, all characteristic peaks that belong to PDPA were observed: $\mathrm{C}=\mathrm{O}$ bonding at $1730 \mathrm{~cm}^{-1}, \mathrm{C}-\mathrm{H}$ vibration peaks at 2948, 2920, and 2890 


\section{Submitted to \\ ADVANCED}

$\mathrm{cm}^{-1}$ and the $\mathrm{C}-\mathrm{N}$ stretching vibration at $1025 \mathrm{~cm}^{-1}$ were assigned to the 2-diisopropyl amino group. The amide I peak at $1660 \mathrm{~cm}^{-1}$ in the H4R4 was observed only in the H4R4-PDPA NPs, indicating successful incorporation of the peptide. For PDPA-NPs and H4R4 (3.0 wt\%)-PDPA-NPs, the PEG-Dox encapsulation efficiency was calculated to be $70 \%$ and $40 \%$ respectively, while the loading efficiency was calculated to be 56\% and 32\% respectively (see Experimental Section).
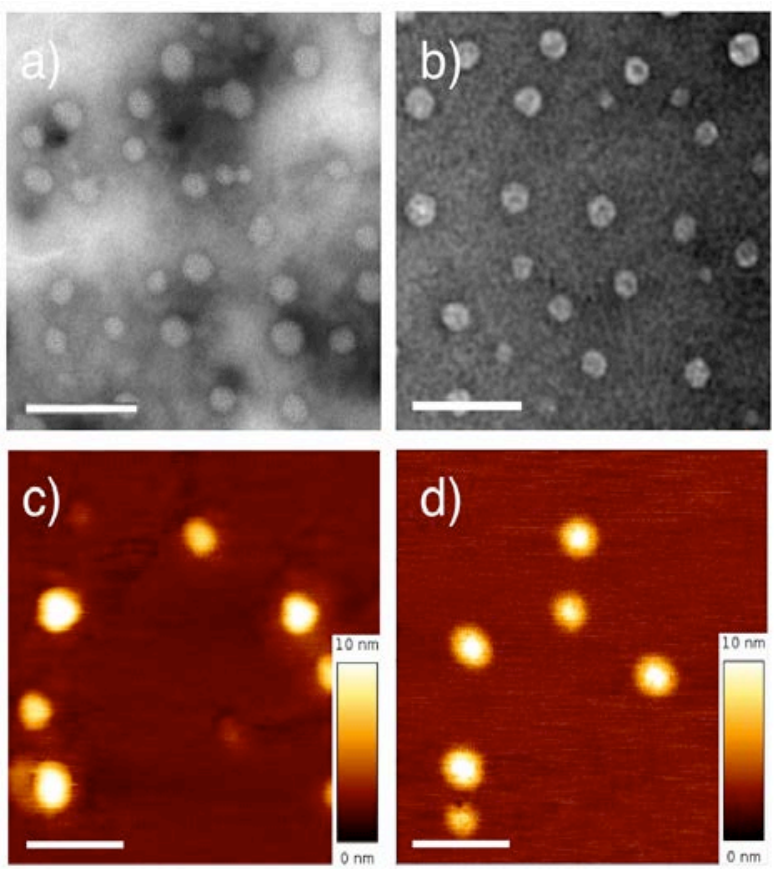

Figure 1. TEM images of air-dried a) PDPA NPs and b) H4R4 (3.0 wt\%)-PDPA NPs dispersed in $\mathrm{pH} 7.4$ PBS. Scale bars are $300 \mathrm{~nm}$. AFM images of air-dried c) PDPA NPs and d) H4R4 (3.0 wt\%)-PDPA NPs dispersed in pH 7.4 PBS. Scale bars are 200 $\mathrm{nm}$.

To demonstrate the pH-induced release of cargo, the Dox release kinetics from the NPs was investigated at both the extracellular $\mathrm{pH}$ of 7.4 and the endosomal $\mathrm{pH}$ of 5.5. ${ }^{[10]}$ At $\mathrm{pH} 7.4$, less than $10 \%$ of Dox was released within 36 h, suggesting that the NPs are largely stable at extracellular $\mathrm{pH}$; in contrast, at $\mathrm{pH} 5.5$, over $90 \%$ of the loaded Dox was released within $36 \mathrm{~h}$ from both the PDPA NPs and H4R4-PDPA NPs, due to the disassembly of the NPs upon protonation of PDPA (Figure 2). The minor hydrophobic DPA moieties at pH 5.5 could interact with the hydrophobic portion of 
PEG-Dox, slowing down the release at this $\mathrm{pH}$. Both NPs demonstrated similar drug release profiles, indicating that the H4R4 did not influence the release of the drug. Furthermore, this $\mathrm{pH}$ sensitive release could be applicable to a wide range of poorly water-soluble drugs, without the need for complicated chemical modifications.

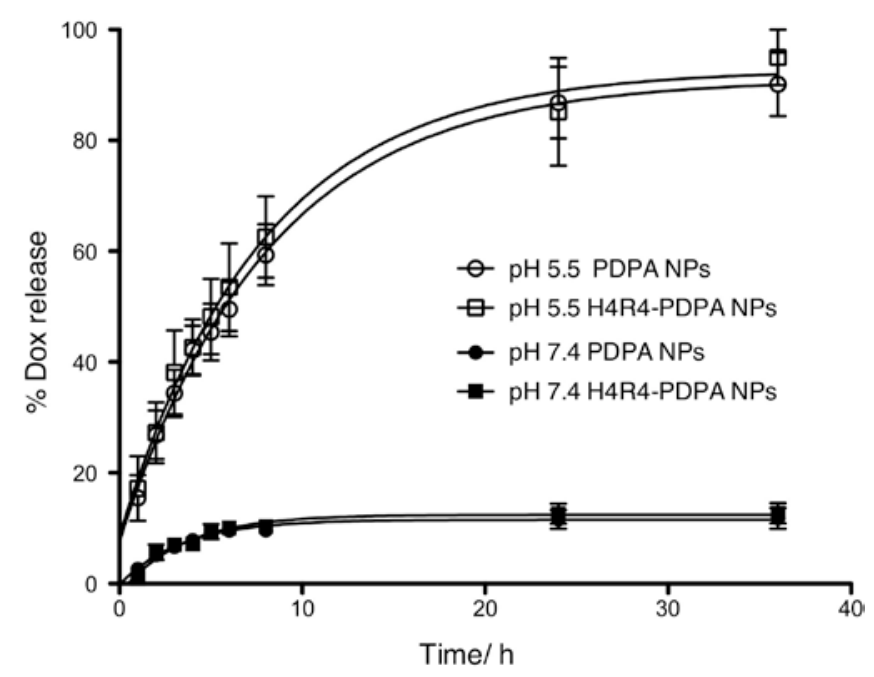

Figure 2. In vitro release profiles of the PEG-Dox conjugate from PDPA NPs and H4R4-PDPA NPs in PBS (pH 5.5 and $\mathrm{pH}$ 7.4). Experiments were performed in triplicate.

The cytotoxic effect of the NPs was examined against HeLa cells, which are a wellestablished cervical cancer cell line. Cell viability was evaluated after NP exposure for 36 h by a 3-(4,5-dimethylthiazol-2-yl)-2,5-diphenyltetrazolium bromide (MTT) assay (Figure 3). PDPA NPs were less cytotoxic than free Dox, which is common in micelle and NP systems. ${ }^{[14]}$ Interestingly, the loading of H4R4 (3.0 wt\%) significantly augmented the cytotoxic effect of the NPs. The free H4R4 peptide, as well as PDPA, had no effect on cell viability, demonstrating that the cytotoxicity of the NPs is due to Dox (Supporting Information, Figure S4b). The mixed solution of H4R4 and PEGDox did not lead to enhanced cytotoxicity (Supporting Information, Figure S4a), suggesting that the incorporation of H4R4 into the NPs plays an important role in 


\section{Submitted to

enhancing the cytotoxicity of PEG-Dox. Furthermore, tunable peptide-facilitated cytotoxicity gating was achieved by simply varying the amount of loaded peptide (0.7, 3.0, or $14 \mathrm{wt} \%)$. The resultant NPs all demonstrated similar size and morphology (Supporting Information, Figure S5). Increasing cytotoxicity was observed from the NPs with increasing amounts of H4R4 (Figure 3b). The $\mathrm{IC}_{50}$ (i.e., inhibitory concentration to produce $50 \%$ cell death) values were determined to be $0.063,0.16$, 0.43, 1.0, and 2.0 $\mu \mathrm{M}$ Dox for H4R4 (14 wt\%)-, H4R4 (3.0 wt\%)-, H4R4 (0.7 wt\%)PDPA NPs, free Dox, and PDPA NPs, respectively (Figure 3). Compared with similar self-assembled delivery systems (Supporting Information, Table S1), the reported IC $_{50}$ values are within a similar range for those for PDPA NPs. However, the co-delivery of peptide and drug enhanced the cytotoxicity by up to a factor of 30. This highlights that the efficiency of NPs against the cancer cells could be augmented (between 3and 30-fold) by incorporating varying amounts of H4R4.

To investigate the mechanism for enhanced cytotoxicity of H4R4-PDPA NPs HeLa cells were treated with the NPs and the kinetics of cellular uptake were evaluated by monitoring the fluorescence of Dox by flow cytometry. Similar rates of cellular uptake were observed, irrespective of the loading of H4R4 (Supporting Information, Figure S6). The uptake profiles were consistent with the similar zeta-potentials measured for PDPA NPs and H4R4-PDPA NPs ( $-5 \pm 3 \mathrm{mV}$ and $-4 \pm 3 \mathrm{mV}$, respectively). These data suggest that the loading of H4R4 has negligible influence on the surface properties and cellular uptake of the NPs. The intracellular distribution of the NPs was monitored in HeLa cells by deconvolution microscopy. In the absence of H4R4, the PEG-Dox mainly accumulated in the cytoplasm and overlapped with the lysosomal-associated membrane protein 1 (LAMP1), suggesting that entrapment in the endosome/lysosome limited the diffusion of PEG-Dox (Figure 4a-e, and 


\section{Submitted to

Supporting Information Figures S7). Meanwhile, a diffuse Dox signal was observed in the nucleus of the cells treated with H4R4-PDPA NPs (Figure $4 \mathrm{f}-\mathrm{j}$ ). These images along with colocalization data between PEG-Dox and endosomes or lysosomes (Figure 4 e,j, Supporting Information, Figures S7e, j) supports the notion that the codelivery of H4R4 facilitated endosomal/lysosomal escape and nuclear translocation of the drug.
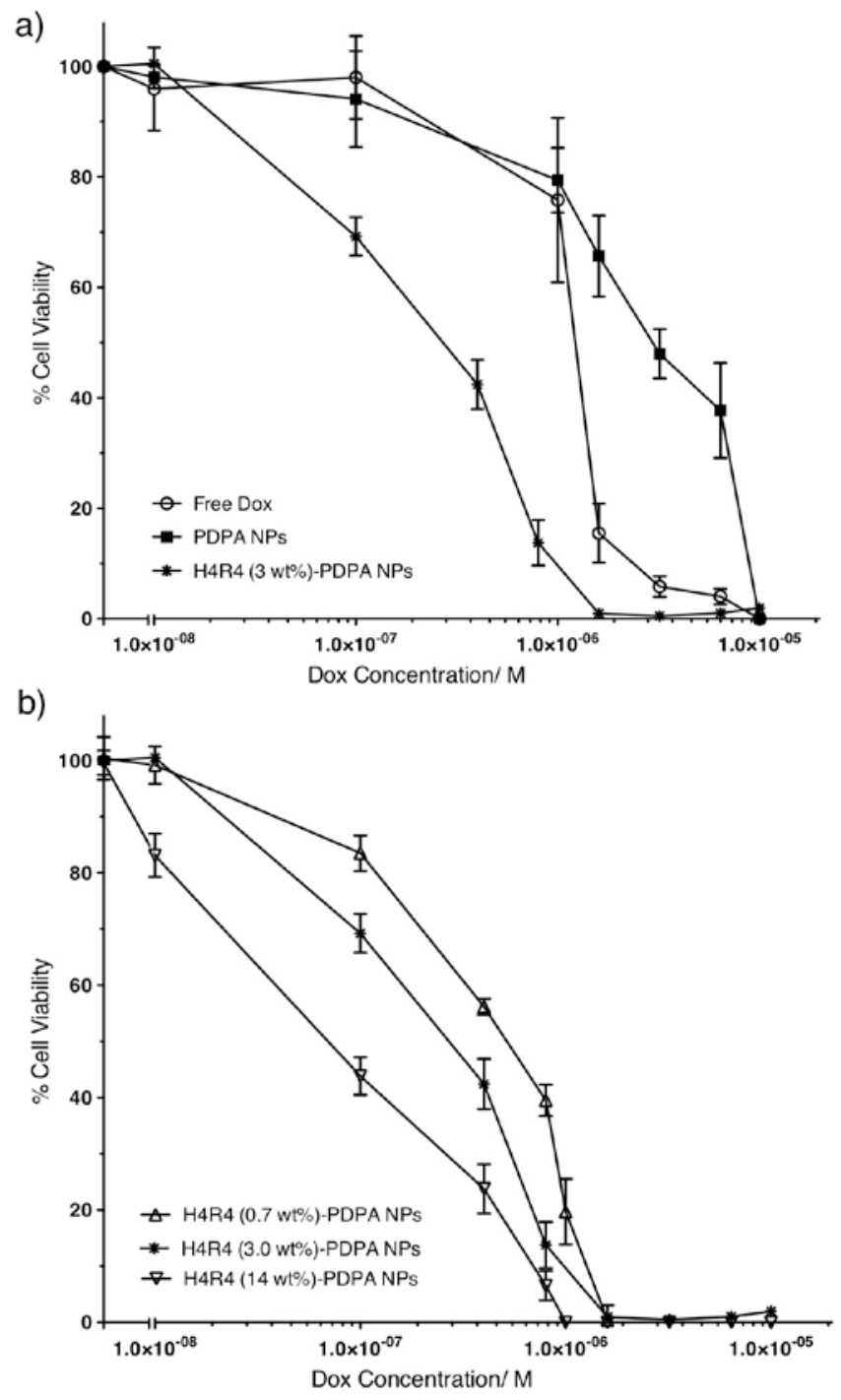

Figure 3. Cell viability assay performed on HeLa cells in the presence of the PDPA NPs and H4R4-PDPA NPs. Cell viability was measured by MTT assays after $36 \mathrm{~h}$ incubation at $37^{\circ} \mathrm{C}$. Data show the normalized mean and standard error of three independent experiments, each performed in triplicate. 


\section{Submitted to

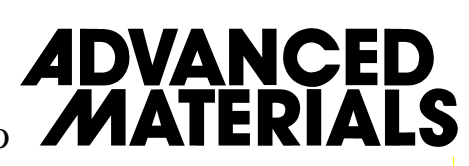

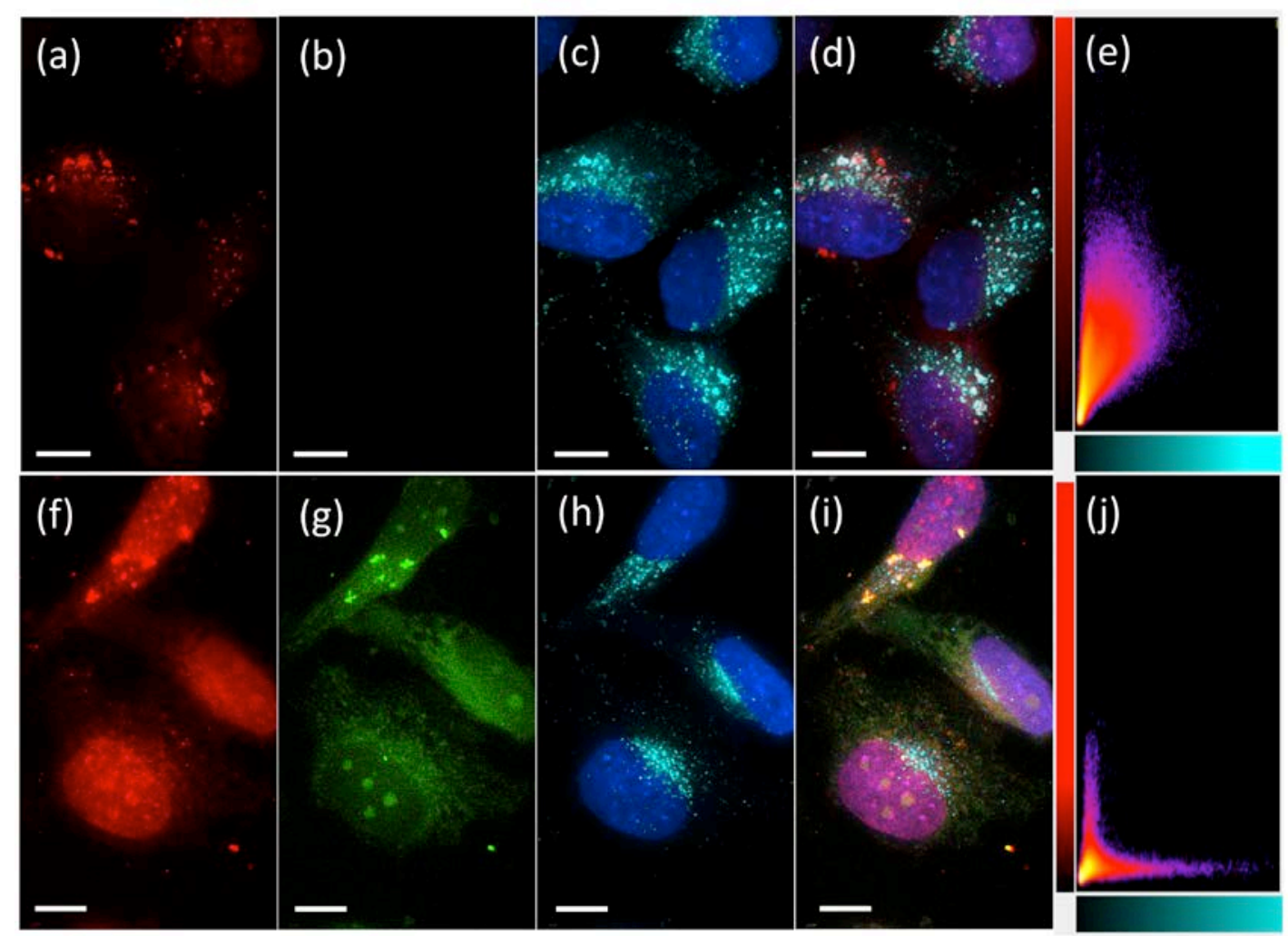

Figure 4. Deconvolution optical microscopy images (maximum intensity projection) of HeLa cells incubated with a-e) PDPA NPs and f-j) H4R4-PDPA NPs for $12 \mathrm{~h}$. a, f) PEG-Dox, g) H4R4, c, h) nucleus, late endosome and lysosome, d, i) overlay, e, j) colocalization analysis between Dox and LAMP1, calculated from individual fluorescence points. PEG-Dox (red), H4R4 peptide (green), DAPI (blue) and lysosomal-associated membrane protein 1 (LAMP1) (cyan). The fluorescence intensity (a-d, f-i) does not allow for direct comparison due to different instrument settings used. Scale bars are $10 \mu \mathrm{m}$.

In conclusion, multifunctional NPs were synthesized in one-step by mixing several simple building blocks. The resultant NPs possessed a pH trigger to simultaneously lock/release anticancer drugs and peptides. We have demonstrated tunable cytotoxicity through non-toxic peptide-facilitated gating. This simple and versatile assembly technique will allow diverse hydrophobic drugs/diagnostic molecules to be easily incorporated, making it attractive for a wide range of biomedical applications.

\section{Experimental Section}




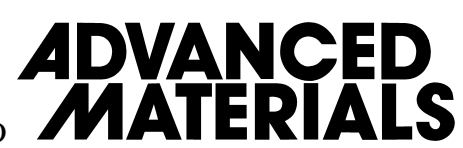

Materials: 2-diisopropylaminoethyl methacrylate (DPA) was purchased from Polysciences, Inc. and passed over basic alumina (Scharlau) twice to remove inhibitors and stored below $-20{ }^{\circ} \mathrm{C}$ prior to use. Ethyl 2-bromoisobutyrate (EBIB), tetra-n-butyl ammonium fluoride (1.0 M in tetrahydrofuran, THF), and $\mathrm{N}, \mathrm{N}, \mathrm{N}^{\prime}, \mathrm{N}^{\prime}, \mathrm{N}^{\prime \prime}-$ pentamethyldiethylenetriamine (PMDETA) were obtained from Sigma-Aldrich and used without further purification. Hydroxy polyethylene glycol Nhydroxysuccinimide ester (PEG-NHS, $\mathrm{M}_{\mathrm{w}}$ 2000) was obtained from JenKem Technology USA. Doxorubicin hydrochloride (Dox) was obtained from Ochem incorporation USA. $\mathrm{H}_{2} \mathrm{~N}-\mathrm{HHHHRRRR-COOH}$ (H4R4) was purchased from Eurogentech. All other materials were obtained from Sigma-Aldrich and used as received.

Synthesis of poly(2-diisopropylaminoethyl methacrylate) (PDPA): PDPA was prepared by atom transfer radical polymerization (ATRP). DPA (1 g, $4.69 \mathrm{mmol}$ ), EBIB (9.1 mg, $0.05 \mathrm{mmol}$ ), PMDETA (24 mg, $0.14 \mathrm{mmol}$ ), and isopropanol (2 mL) were placed into a Schlenk flask equipped with a magnetic stir bar. The flask was degassed via three freeze-thaw-pump cycles and back-filled with argon. $\mathrm{CuCl}$ (5 mg, $0.05 \mathrm{mmol}$ ) was then introduced into the reaction flask to start the polymerization at $23{ }^{\circ} \mathrm{C}$. After $16 \mathrm{~h}$, the polymerization was terminated by exposing the reaction solution to air and diluting with THF (50 mL) leading to the aerial oxidation of the $\mathrm{Cu}(\mathrm{I})$ catalyst. The solution was then passed through a neutral alumina column using THF as the eluent to remove the copper catalyst. The collected eluent was concentrated and precipitated into cold $n$-hexane. This purification cycle was repeated twice. The resulting product was dried in vacuo to yield PDPA as a colorless viscous solid (0.90 g). ${ }^{1} \mathrm{H}-\mathrm{NMR}\left(400 \mathrm{MHz}, \mathrm{CDCl}_{3}\right)$ : ( $\delta$, ppm) 4.1-3.6 (-COO-CH$-\mathrm{CH}_{2}$ ), 3.1- 
$2.85\left(\mathrm{CH}_{2}-\mathrm{CH}_{2}-\mathrm{N}\right)$, 2.7-2.5 (N-CH( $\left.\left(\mathrm{CH}_{3}\right)_{2}\right)$, 2.1-0.5 (backbone). GPC-MALLS (THF): $M_{\mathrm{w}}=10.7 \mathrm{kDa}, M_{\mathrm{w}} / M_{\mathrm{n}}=1.12$.

Conjugation of Dox-PEG: PEG-NHS (10 mg, 5 mmol), Dox (4.35 mg, $7.5 \mathrm{mmol}$ ), and triethylamine $(1.25 \mu \mathrm{L}, 9 \mathrm{mmol})$ was dissolved in methanol $(10 \mathrm{~mL})$. The resulting solution was stirred at $23{ }^{\circ} \mathrm{C}$ for $2 \mathrm{~h}$ and then dialyzed against deionized water more than ten times $(\mathrm{MW}$ cutoff $=2 \mathrm{~K}$, Spectra/Por 6$)$ and freeze dried.

Preparation of PDPA NPs: 2 mg Dox-PEG and $1 \mathrm{mg}$ PDPA was dissolved in $0.3 \mathrm{~mL}$ ethanol. For the H4R4-loaded particles, $0.2 \mathrm{mg}$ (0.7 wt\%), $0.1 \mathrm{mg}$ (3 wt\%) and 0.5 mg (14 wt\%) H4R4 was added in the above mentioned solution mixture. This solution was then added drop-by-drop to $3 \mathrm{~mL}$ PBS while vigorous stirring. The organic solvent was evaporated while being stirred for $48 \mathrm{~h}$. The remaining solution was filtered through a $0.45 \mu \mathrm{m}$ pore size filter to remove any polymer aggregates and then dialyzed against PBS more than ten times to remove free polymers and peptides in the solution (MW cutoff $=10$ 000, Thermo Scientific).

Characterization of PDPA NPs: The size of PDPA NPs was determined using a Malvern Zeta Sizer NanoZS (Malvern Instruments, UK) measuring at a fixed scattering angle of $173^{\circ}$. The morphology of the particles was analyzed using transmission electron microscopy (TEM) and atomic force microscopy (AFM). TEM images were taken by Philips CM120 BioTWIN TEM with an operation voltage of $120 \mathrm{kV}$. Samples of NPs were air-dried on a carbon-coated Formvar film mounted on 300 mesh copper grids (ProSciTech, Australia). Negative staining was achieved by adding a drop of phosphotungstic acid $(1 \mathrm{mg} / \mathrm{mL})$ in deionized water. For AFM measurements, particle solutions air-dried on silica wafers were mounted on a NanoWizard II AFM (JPK Instruments, Berlin, Germany) in intermittent contact mode with MikroMasch silicon cantilevers (NSC/CSC). Fourier transform infrared 


\section{ADVANCED}

(FTIR) spectra were recorded on a Varian 7000 FTIR spectrometer with a Specac

MKII Golden Gate single-reflectance diamond ATR attachment with KRS-5 optics and a heated top plate maintained at $30^{\circ} \mathrm{C}$. Polymer particle solutions were dropped and dried onto the diamond ATR crystal, and spectra were collected in the absorbance mode from 4000 to $400 \mathrm{~cm}^{-1}$ at a resolution of $2 \mathrm{~cm}^{-1}$. The PEG-Dox encapsulation efficiency was calculated by using the weight of PEG-Dox in the filtered NPs and the total weight of PEG-Dox added, while the loading efficiency was calculated using the weight of PEG-Dox in the filtered NPs and the weight of the freeze-dried filtered NPs. The weight of PEG-Dox loaded in the filtered NPs was determined from a calibration curve by disassembling the NPs and diluting the solution appropriately.

In vitro release profile: $1 \mathrm{~mL}$ of the PDPA NP or H4R4-PDPA NP dispersion was placed into a dialysis device (Slide-A-Lyzer MINI, MW cutoff $=10 \mathrm{~K}$, Thermo Scientific) placed in a tube equipped with a magnetic stirring bar with $50 \mathrm{~mL}$ PBS at different $\mathrm{pH}$ values under constant stirring. At each time interval, $180 \mu \mathrm{L}$ of the PBS was taken and measured under fluorescence spectrophotometer. The solutions were excited at $470 \mathrm{~nm}$ and emissions at $560 \mathrm{~nm}$ were determined. When the concentration of the PEG-Dox was diluted between $0-0.0005 \mathrm{mg} / \mathrm{mL}$, a linear relationship was observed between fluorescence emission and concentration of the PEG-DOX, and this dilution factor was used to assess the release of the drug from the NPS.

Particle-cell association study: HeLa cells were seeded at $37{ }^{\circ} \mathrm{C}\left(5 \% \mathrm{CO}_{2}\right)$ overnight at a population of $2 \times 10^{4}$ cells per well in to a 48 well plate. An aliquot of the PDPA NP or H4R4-PDPA NP dispersion was added in each well and the final Dox concentration was adjusted to $1 \times 10^{-6} \mathrm{M}$. At each time interval, the cells were trypsinized and washed twice with PBS, and then transferred into a Röhren tube for fluorescence intensity examination using flow cytometry. 


\section{Submitted to

Cytotoxicity assay: The 3-(4,5-dimethylthiaol-2-yl)-2,5-diphenyltetrazolium bromide (MTT)-based in vitro cytotoxicity assay was performed to compare anti-cancer effects of the polymer particles against HeLa cell lines according to the previously established method. ${ }^{[15]}$ HeLa cells were seeded on a 96 well plate at a population of 2 $\times 10^{4}$ cells per well. After incubation with PDPA NPs, H4R4-PDPA NPs, Dox-PEG conjugate, PDPA, H4R4 peptide, Dox-PEG conjugate and H4R4 peptide mixture, or free Dox at various concentrations for $36 \mathrm{~h}, 20 \mu \mathrm{L}$ of MTT $(5 \mathrm{mg} / \mathrm{mL})$ was added to each well. Following $2 \mathrm{~h}$ incubation at $37{ }^{\circ} \mathrm{C}\left(5 \% \mathrm{CO}_{2}\right)$, the MTT product was dissolved in isopropanol and the percentage of cell viability was determined from the absorption at $580 \mathrm{~nm}$ relative to non-treated cells.

Deconvolution fluorescence microscopy: Deconvolution fluorescence microscopy was performed on a DeltaVision (Applied Precision) microscope with a $60 \times 1.42$ NA oil objective with a standard FITC/TRITC/CY5 filter set. Images were processed with Imaris (Bitplane) using the maximum intensity projection. 


\section{Submitted to

\section{Supporting Information}

Supporting Information is available online from the Wiley Online Library or from the author.

\section{Acknowledgements}

This work was supported by Australian Research Council under the Australian Laureate Fellowship FL120100030 (F.C.), Discovery Project DP0877360 (F.C.), Super Science Fellowship FS110200025 (J.C. and F.C.), and Australian Future Fellowship FT120100564 (G.K.S.) schemes.

Received: ((will be filled in by the editorial staff))

Revised: ((will be filled in by the editorial staff)) Published online: ((will be filled in by the editorial staff))

[1] a) V. P. Torchilin, Nat. Rev. Drug Discov. 2005, 4, 145; b) D. C. Drummond, O. Meyer, K. Hong, D. B. Kirpotin, D. Papahadjopoulos, Pharmacol. Rev. 1999, 51, 691; c) T. Lammers, W. E. Hennink, G. Storm, Br. J. Cancer 2008, 99, 392.

[2] a) Y. Wang, S. Gao, W.-H. Ye, H. S. Yoon, Y.-Y. Yang, Nat. Mater. 2006, 5, 791; b) M. Khan, Z. Y. Ong, N. Wiradharma, A. B. E. Attia, Y.-Y. Yang, Adv. Healthcare Mater. 2012, 1, 373; c) S. Chen, D. Zhao, F. Li, R.-X. Zhuo, S.-X. Cheng, RSC Adv. 2012, 2, 1820; d) M. Creixell, N. A. Peppas, Nano Today 2012, 7, 367.

[3] a) I. Nakase, Y. Konishi, M. Ueda, H. Saji, S. Futaki, J. Controlled Release 2012, 159, 181; b) V. P. Torchilin, Pept. Sci. 2008, 90, 604; c) N. Ferrer-Miralles, E. Vázquez, A. Villaverde, Trends Biotechnol. 2008, 26, 267-275; d) S. Futaki, Pept. Sci. 2006, 84, 241; e) Z. Poon, D. Chang, X. Zhao, P. T. Hammond, ACS Nano 2011, 5, 4284.

[4] a) E. Vivès, J. Schmidt, A. Pèlegrin, Biochim. Biophys. Acta, Rev. Cancer 2008, 1786, 126; b) J. Regberg, A. Srimanee, Ü. Langel, Pharmaceuticals 2012, 5, 991; c) E. Vázquez, N. Ferrer-Miralles, A. Villaverde, Drug Discovery Today 2008, 13, 1067; d) F. Heitz, M. C. Morris, G. Divita, Br. J. Pharmacol. 2009, 157, 195. 
Submitted to

\section{ADNANCES
MAAERTALIS}

[5] K. Melikov, L. V. Chernomordik, Cell. Mol. Life Sci. 2005, 62, 2739.

[6] V. A. Sethuraman, Y. H. Bae, J. Controlled Release 2007, 118, 216.

[7] a) B. Langlet-Bertin, C. Leborgne, D. Scherman, B. Bechinger, A. J. Mason,

A. Kichler, Pharm. Res. 2010, 27, 1426; b) P. Midoux, A. Kichler, V. Boutin, J.-C. Maurizot, M. Monsigny, Bioconjugate Chem. 1998, 9, 260.

[8] D. Putnam, C. A. Gentry, D. W. Pack, R. Langer, Proc. Natl. Acad. Sci. U.S.A. 2001, 98, 1200.

[9] a) J. Du, S. P. Armes, J. Am. Chem. Soc. 2005, 127, 12800; b) K. Liang, G. K.

Such, Z. Zhu, Y. Yan, H. Lomas, F. Caruso, Adv. Mater. 2011, 23, H273.

[10] J. R. Casey, S. Grinstein, J. Orlowski, Nat. Rev. Mol. Cell Biol. 2010, 11, 50.

[11] K. Liang, G. K. Such, Z. Zhu, S. J. Dodds, A. P. R. Johnston, J. Cui, H. Ejima, F. Caruso, ACS Nano 2012, 6, 10186.

[12] D. E. Discher, A. Eisenberg, Science 2002, 297, 967.

[13] V. L. Jaspreet K. Vasir, Technol. Cancer Res. Treat. 2005, 4, 363.

[14] F. M. Veronese, O. Schiavon, G. Pasut, R. Mendichi, L. Andersson, A. Tsirk, J. Ford, G. Wu, S. Kneller, J. Davies, R. Duncan, Bioconjugate Chem. 2005, 16, 775.

[15] T. Mosmann, J. Immunol. Methods 1983, 65, 55-63. 


\section{Submitted to \\ ADVANCED}

A novel class of nanoparticles is developed for the co-delivery of a short cell penetrating peptide and chemotherapeutic drugs to achieve enhanced cytotoxicity. Tunable cytotoxicity is achieved through non-toxic peptide-facilitated gating. The strategy relies on a one-step blending process from polymer building blocks to form monodisperse, PEGylated particles that are sensitive to cellular $\mathrm{pH}$ variations. By varying the amount of peptide loading, the chemotherapeutic effects can be enhanced by up to 30 -fold.

Keyword self-assembly, co-delivery, drug delivery, peptide, biomaterials

K. Liang, J. J. Richardson, H. Ejima, G. K. Such, J. Cui, F. Caruso*

Peptide-Tunable Drug Cytotoxicity via One-Step Assembled Polymer Nanoparticles

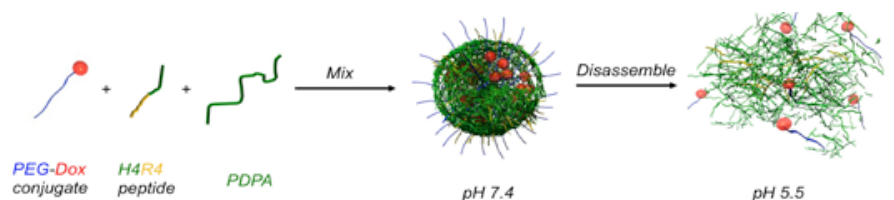




\section{University Library}

\section{- M M N E R VA A gateway to Melbourne's research publications}

Minerva Access is the Institutional Repository of The University of Melbourne

Author/s:

Liang, K;Richardson, JJ;Ejima, H;Such, GK;Cui, J;Caruso, F

Title:

Peptide-Tunable Drug Cytotoxicity via One-Step Assembled Polymer Nanoparticles

Date:

2014-04-01

Citation:

Liang, K., Richardson, J. J., Ejima, H., Such, G. K., Cui, J. \& Caruso, F. (2014). PeptideTunable Drug Cytotoxicity via One-Step Assembled Polymer Nanoparticles. ADVANCED MATERIALS, 26 (15), pp.2398-2402. https://doi.org/10.1002/adma.201305002.

Persistent Link:

http://hdl.handle.net/11343/123279 